\title{
Probing trilinear gauge boson interactions via single electroweak gauge boson production at the CERN LHC
}

\author{
O. J. P. Éboli* \\ Instituto de Física da USP, C.P. 66.318, São Paulo, SP 05315-970, Brazil \\ M. C. Gonzalez-Garcia ${ }^{\dagger}$ \\ Y.I.T.P., SUNY at Stony Brook, Stony Brook, New York 11794-3840, USA \\ IFIC, Universitat de València - C.S.I.C., Apartado 22085, 46071 València, Spain
}

(Received 7 June 2004; published 13 October 2004)

\begin{abstract}
We analyze the potential of the CERN Large Hadron Collider (LHC) to study anomalous trilinear vector-boson interactions $W^{+} W^{-} \gamma$ and $W^{+} W^{-} Z$ through the single production of electroweak gauge bosons via the weak boson fusion processes $q q \rightarrow q q W\left(\rightarrow \ell^{ \pm} \nu\right)$ and $q q \rightarrow q q Z\left(\rightarrow \ell^{+} \ell^{-}\right)$with $\ell=e$ or $\mu$. After a careful study of the standard model backgrounds, we show that the single production of electroweak bosons at the LHC can provide stringent tests on deviations of these vertices from the standard model prediction. In particular, we show that single gauge-boson production exhibits a sensitivity to the couplings $\Delta \kappa_{Z, \gamma}$ similar to that attainable from the analysis of electroweak boson pair production.
\end{abstract}

DOI: 10.1103/PhysRevD.70.074011

PACS numbers: 12.38.Bx

\section{INTRODUCTION}

Within the framework of the standard model (SM), the structure of the trilinear and quartic vector-boson couplings is completely determined by the $\mathrm{SU}(2)_{L} \times \mathrm{U}(1)_{Y}$ gauge symmetry. The study of these interactions can either lead to an additional confirmation of the model or give some hint on the existence of new phenomena at a higher scale [1]. The triple gauge-boson vertices (TGV's) have been probed directly at the Tevatron [2] and LEP $[3,4]$ through the production of vector-boson pairs, and the experimental results agree with the SM predictions within $\mathcal{O}(10 \%)$; see Table I. Moreover, TGV's contribute at the one-loop level to the $Z$ physics and consequently they can also be indirectly constrained by precision electroweak data [5]. At the LHC, the TGV's will be subject to a more severe scrutiny via the production of electroweak boson pairs ( $W \gamma$ and $W Z$ ) [7] which will probe these couplings at the few percentage level [6].

In this work we analyze the LHC potential to study the TGV's through the weak boson fusion (WBF) reactions

$$
\begin{aligned}
& p p \rightarrow j j W^{+} j j l^{+} \nu_{l}, \quad p p \rightarrow j j W^{-} \rightarrow j j l^{-} \bar{\nu}_{l}, \\
& p p \rightarrow j j Z \rightarrow j j l^{+} l^{-},
\end{aligned}
$$

with $l=e, \mu$. These processes are complementary to the electroweak gauge-boson pair production in the analysis of the $W W \gamma$ and $W W Z$ vertices; certainly a larger number of basic processes and observables can contribute to a better scrutiny of the TGV's. While $W Z, W^{+} W^{-}$, and $W \gamma$ production at the LHC probe the TGV's for timelike momenta of all vector bosons, the single $W$ and $Z$ pro-

\footnotetext{
*Electronic address: eboli@fma.if.usp.br

†Electronic address: concha@insti.physics.sunysb.edu
}

ductions via WBF present two electroweak gauge bosons with spacelike momentum transfer.

TGV's in single gauge-boson production processes were studied in Ref. [8] for the superconducting supercollider energy but had not been discussed in the context of the LHC. A potential drawback of single gauge-boson production as a test of electroweak vertices at the LHC energies is the large expected background from higher order QCD corrections to the Drell-Yan process [9]. ${ }^{1}$ However, it has recently been proved in the case of Higgs production [11] that these backgrounds are under control due to the presence of two very energetic forward jets. Furthermore, its theoretical uncertainty can be efficiently reduced by making use of a calibration region where the backgrounds can be estimated from data. In this work we show that, indeed, these conclusions apply to the study of TGV's in single gauge-boson production.

In the following, we describe the $W^{+} W^{-} V(V=Z$ or $\gamma$ ) vertices in terms of the standard Lorentz invariant and $C P$ conserving parametrization, which is given by the effective Lagrangian [12]

$$
\begin{aligned}
\mathcal{L}_{\mathrm{eff}}^{W W V}= & -i g_{W W V}\left[g_{1}^{V}\left(W_{\mu \nu}^{+} W^{-\mu}-W_{\mu \nu}^{-} W^{+\mu}\right) V^{\nu}\right. \\
& +\kappa_{V} W_{\mu}^{+} W_{\nu}^{-} V^{\mu \nu}+\frac{\lambda_{V}}{M_{W}^{2}} W_{\mu}^{+\nu} W_{\nu}^{-\rho} V_{\rho}^{\mu} \\
& \left.-i g_{5}^{V} \epsilon^{\mu \nu \rho \sigma}\left(W_{\mu}^{+} \partial_{\rho} W_{\nu}^{-}-W_{\nu}^{-} \partial_{\rho} W_{\mu}^{+}\right) V_{\sigma}\right]
\end{aligned}
$$

where $V_{\mu \nu}=\partial_{\mu} V_{\nu}-\partial_{\nu} V_{\mu}, g_{W W \gamma}=e$, and $g_{W W Z}=$ $e c_{W} / s_{W}$, with $s_{W}\left(c_{W}\right)=\sin (\cos ) \theta_{W}$. The first three terms

\footnotetext{
${ }^{1}$ Conversely, QCD corrections to the electroweak production have been shown to be modest [10].
} 
TABLE I. 95\% CL limits on the anomalous couplings emanating from direct measurements at LEP2 and from loop contributions to the precision measurements at LEP I [5], assuming the HISZ scenario $\Delta \kappa_{\gamma}=\left(c_{W}^{2} / s_{W}^{2}\right)\left(\Delta g_{1}^{Z}-\Delta \kappa_{Z}\right)$ and $\lambda_{Z}=\lambda_{\gamma}$. We also present the expected $95 \%$ CL bounds at the LHC obtained through the pair production of electroweak gauge bosons [6], assuming a form factor $1 /\left(1+\hat{s} / \Lambda^{2}\right)^{2}$ with $\Lambda=10 \mathrm{TeV}$ and $\hat{s}$ being the squared partonparton center-of-mass energy. The entry marked as $\cdots$ has not been evaluated in the literature.

\begin{tabular}{ccccc}
\hline \hline Anomalous coupling & Direct LEP limits & Indirect limits & Pair production limits at the LHC \\
\hline$\Delta \kappa_{\gamma}$ & {$[-0.105,0.069]$} & {$[-0.044,0.059]$} & {$[-0.034,0.034]$} \\
$\lambda_{\gamma}$ & {$[-0.059,0.026]$} & {$[-0.061,0.10]$} & {$[-0.0014,0.0014]$} \\
$g_{1}^{Z}$ & {$[-0.051,0.034]$} & {$[-0.051,0.0092]$} & {$[-0.0038,0.0038]$} \\
$\Delta \kappa_{Z}$ & {$[-0.040,0.046]$} & {$[-0.050,0.0039]$} & {$[-0.040,0.040]$} \\
$\lambda_{Z}$ & {$[-0.059,0.026]$} & {$[-0.061,0.10]$} & {$[-0.0028,0.0028]$} \\
$g_{5}^{Z}$ & {$[-0.95,0.079]$} & {$[-0.085,0.049]$} & $\ldots$ \\
\hline \hline
\end{tabular}

in Eq. (2) are $C$ and $P$ invariant, while the last one violates both $C$ and $P$. Electromagnetic gauge invariance implies that $1-g_{1}^{\gamma}=g_{5}^{\gamma}=0$. Within the framework of the SM, $g_{1}^{\gamma}=g_{1}^{Z}=\kappa_{\gamma}=\kappa_{Z}=1 \quad$ and $\lambda_{\gamma}=\lambda_{Z}=g_{5}^{Z}=0$.

Since the standard model is consistent with the available experimental data, it is natural to parametrize the anomalous TGV's in terms of an effective Lagrangian which exhibits the $\mathrm{SU}(2)_{L} \times \mathrm{U}(1)_{Y}$ gauge invariance. The particular way this symmetry is realized depends on the particle content at low energies. If the spectrum at low energies does not exhibit a light Higgs boson, this symmetry has to be nonlinearly realized and the triple gauge-boson vertex can be parametrized as Eq. (2) with the couplings $g_{1}^{Z}, \kappa_{\gamma}, \kappa_{Z}, \lambda_{\gamma}, \lambda_{Z}$, and $g_{5}^{Z}$ being independent parameters [13].

Conversely, if a light Higgs boson is present, the symmetry can be realized linearly [14-16]. In this case, the leading effects of new interactions are described by 11 dimension-six operators $\mathcal{O}_{i}$,

$$
\mathcal{L}_{\text {eff }}^{\text {linear }}=\sum_{i} \frac{f_{i}}{\Lambda^{2}} \mathcal{O}_{i}
$$

at energies below the new physics scale $\Lambda$. Three of these operators [16], namely,

$$
\begin{aligned}
\mathcal{O}_{B} & =\left(D_{\mu} \Phi\right)^{\dagger} \hat{B}^{\mu \nu}\left(D_{\nu} \Phi\right), \\
\mathcal{O}_{W} & =\left(D_{\mu} \Phi\right)^{\dagger} \hat{W}^{\mu \nu}\left(D_{\nu} \Phi\right), \\
\mathcal{O}_{W W W} & =\operatorname{Tr}\left[\hat{W}_{\mu \nu} \hat{W}^{\nu \rho} \hat{W}_{\rho}^{\mu}\right],
\end{aligned}
$$

modify the triple gauge-boson couplings without affecting the gauge-boson two-point functions at tree level, the so-called "blind" operators. In our notation, $\hat{B}_{\mu \nu}=$ $i\left(g^{\prime} / 2\right) B_{\mu \nu}$ and $\hat{W}_{\mu \nu}=i(g / 2) \sigma^{a} W_{\mu \nu}^{a}$, with $B_{\mu \nu}$ and $W_{\mu \nu}^{a}$ being the $\mathrm{U}(1)_{Y}$ and $\mathrm{SU}(2)_{L}$ full field strengths and $\sigma^{a}$ representing the Pauli matrices. In this framework, it is expected that $g_{5}^{Z}$ should be suppressed since it is related to a dimension-eight operator [17].
The anomalous couplings of the parametrization (2) are related to the coefficients of the linearly realized effective Lagrangian by

$$
\begin{gathered}
\Delta g_{1}^{Z}=f_{W} \frac{m_{Z}^{2}}{2 \Lambda^{2}} \\
\Delta \kappa_{Z}=\left[f_{W}-s_{W}^{2}\left(f_{B}+f_{W}\right)\right] \frac{m_{Z}^{2}}{2 \Lambda^{2}}, \\
\lambda_{Z}=f_{W W W} \frac{3 m_{W}^{2} g^{2}}{2 \Lambda^{2}} .
\end{gathered}
$$

It is interesting to notice that these effective operators lead to the following relation between the coefficients of Lagrangian (2), defining the Hagiwara-IshiharaSzalapski-Zeppenfeld (HISZ) scenario [15]:

$$
\begin{gathered}
\Delta \kappa_{\gamma}=\frac{c_{W}^{2}}{s_{W}^{2}}\left(\Delta g_{1}^{Z}-\Delta \kappa_{Z}\right), \\
\lambda_{\gamma}=\lambda_{Z} .
\end{gathered}
$$

\section{CAlculational tools}

We are considering the production of electroweak gauge bosons $W^{ \pm}$and $Z$ in WBF, $q q \rightarrow q q V^{*} V^{*} \rightarrow q q V$ $(V=W$ or $Z)$, with subsequent decays $Z \rightarrow \ell^{+} \ell^{-}$or $W^{ \pm} \rightarrow \ell^{ \pm} \nu_{\ell}$ with $\ell=e$ or $\mu$. The signal is thus characterized by two quark jets, which typically enter in the forward and backward regions of the detector and are widely separated in pseudorapidity, and by two charged leptons or a charged lepton accompanied by a large transverse momentum imbalance. Significant backgrounds to the anomalous signal arise from $Z j j$ and $W j j$ production, which can take place via standard electroweak subprocesses (including both the WBF and the emission of the electroweak gauge boson from a quark line) and most copiously through Drell-Yan gauge-boson production associated with further real emission. Another potential background is the QCD production of 
top quark pairs, with at least one top quark decaying semileptonically.

The signal and backgrounds were simulated at the parton level with full tree-level matrix elements. This was accomplished by numerically evaluating helicity amplitudes for all subprocesses. Backgrounds include all order $\alpha_{s}^{2}$ real emission corrections to Drell-Yan production, to be called QCD $W j j$ and $Z j j$ processes, and cross sections are calculated with code based on Ref. [18]. The second large classes of processes are the anomalous signal and the electroweak background; this last one denoted by electroweak (EW) $W j j$ and $Z j j$ production. The code for these processes is based on Ref. [19]. Madgraph [20] code was also used to simulate the QCD $t \bar{t}$ background at tree

level. For all QCD effects, the running of the strong coupling constant is evaluated at one-loop order, with $\alpha_{s}\left(M_{Z}\right)=0.12$. We employed CTEQ5L parton distribution functions [21] throughout. We took the electroweak parameters $\sin ^{2} \theta_{W}=0.23124, \alpha_{e m}=1 / 128.93, m_{Z}=$ $91.189 \mathrm{GeV}$, and $m_{W}=79.95 \mathrm{GeV}$, which was obtained imposing the tree-level relation $\cos \theta_{W}=m_{W} / m_{Z}$. We simulate experimental resolutions by smearing the energies (but not directions) of all final state partons with a Gaussian error given by $\Delta(E) / E=0.5 / \sqrt{E} \oplus 0.02$ ( $E$ in $\mathrm{GeV}$ ), while for charged leptons we used a resolution $\Delta(E) / E=0.02 / \sqrt{E}$.

An important feature of the WBF signal is the absence of color exchange between the final state quarks, which leads to a depletion of gluon emission in the region between the two tagging jets. We can enhance the signal to background ratio by vetoing additional soft jet activity in the central region [22]. A central jet veto is ineffective against the EW $W j j$ and $Z j j$ backgrounds, which possess the same color structure as the signal. For the QCD backgrounds, however, there is color exchange in the $t$ channel and consequently a more abundant production of soft jets, with $p_{T}>20 \mathrm{GeV}$, in the central region [19]. The probability of an event to survive such a central jet veto has been analyzed for various processes in Ref. [23], from which we take the veto survival probabilities

$$
P_{\text {surv }}^{\mathrm{EW}}=0.82, \quad P_{\text {surv }}^{\mathrm{QCD}}=0.28,
$$

which are appropriate for the hard tagging jet cuts to be used below.

It is important to note that the operators in Eq. (2) lead to tree-level unitarity violation in $2 \rightarrow 2$ processes at high energies. The standard procedure to avoid this unphysical behavior of the cross section and to obtain meaningful limits is to multiply the anomalous couplings $\left(g_{\text {ano }}^{i}\right)$ by a form factor

$$
g_{\text {ano }}^{i} \rightarrow \frac{g_{\text {ano }}^{i}}{\left[\left(1+\frac{\left|q_{1}^{2}\right|}{\Lambda^{2}}\right)\left(1+\frac{\left|q_{2}^{2}\right|}{\Lambda^{2}}\right)\left(1+\frac{\left|q_{3}^{2}\right|}{\Lambda^{2}}\right)\right]^{n}},
$$

with $q_{i}$ standing for the four-momenta of the gauge bosons in the vertex. Therefore, the choices $n$ and $\Lambda$ are part of the definition the anomalous couplings. In our analysis we chose $\Lambda=2.5 \mathrm{TeV}$ and $n=1$; picking a larger (smaller) value of $n(\Lambda)$ leads to looser bounds on the anomalous TGV's. At $e^{+} e^{-}$colliders the center-ofmass energy is fixed and the introduction of the form factor (11) is basically equivalent to a rescaling of the anomalous couplings; therefore, we should perform this rescaling when comparing results obtained at hadron and $e^{+} e^{-}$colliders. For example, the LEP are weakened by a factor $\simeq 1 \%$ for our choice of $n$ and $\Lambda$.

Altogether, the cross sections for processes (1) can be written as

$$
\sigma=\sigma_{\mathrm{sm}}+\sum_{i=1}^{6} \sigma_{i}^{\mathrm{int}} g_{\mathrm{ano}}^{i}+\sum_{i=1}^{6} \sum_{j \geq i} \sigma_{i j}^{\mathrm{ano}} g_{\mathrm{ano}}^{i} g_{\mathrm{ano}}^{j},
$$

where $\sigma_{\text {sm }}, \sigma_{i}^{\text {int }}$, and $\sigma_{i j}^{\text {ano }}$ are, respectively, the SM cross section, interference between the SM and the anomalous contribution, and the pure anomalous contributions, which contain the interference between the different TGV contributions.

\section{SIGNAL AND BACKGROUND PROPERTIES}

The main features of the production of a single electroweak gauge boson via WBF are the presence of two very energetic forward jets and one or two isolated charged leptons. Therefore, we initially imposed the following jet tagging cuts:

$$
\begin{aligned}
p_{T}^{j} & >40 \mathrm{GeV}, \quad\left|y_{j}\right|<5.0, \\
\left|y_{j 1}-y_{j 2}\right| & >4.4, \quad y_{j 1} y_{j 2}<0,
\end{aligned}
$$

and lepton acceptance and isolation cuts:

$$
\begin{aligned}
\left|y_{\ell}\right| \leq 2.5, & p_{T}^{\ell} \geq 20 \mathrm{GeV}, \\
\Delta R_{\ell j} \geq 0.6, & \Delta R_{\ell \ell} \geq 0.6 .
\end{aligned}
$$

The effect of the jet rapidity separation cut in suppressing the QCD background is illustrated in the upper panels in Fig. 1. We display in the upper left panel of Fig. 1 the rapidity separation between the tagging jets in $W^{+} j j$ production $\left(\left|y_{j 1}-y_{j 2}\right|\right)$ after the cuts (13) and (14) prior to the $\left|y_{j 1}-y_{j 2}\right|>4.4$ cut for both EW and QCD backgrounds; for most variables the anomalous signal presents kinematics distributions similar to the EW background, and, consequently, we show only the EW distribution in these cases. As we can see, the rapidity separation between the tagging jets for the QCD processes peaks at small values, while the EW and signal processes lead to larger rapidity separations. Consequently imposing the rapidity separation cut enhances the EW/QCD ratio by a factor of $\sim 20$. However, further cuts are necessary to reduce the QCD background to acceptable levels. This can be achieved by making use of the differences in the 

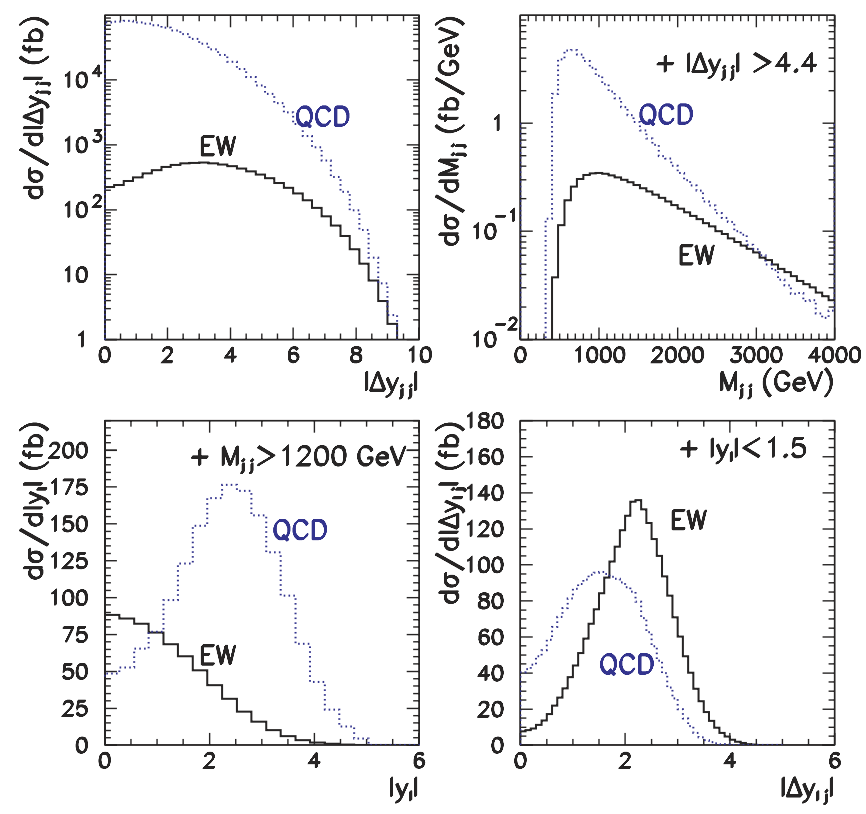

FIG. 1 (color online). Event distributions for EW and QCD contributions to $W^{+} j j$ with $W^{+} \rightarrow l^{+} \nu_{l}$ at the LHC. In the left upper panel, only the cuts in Eqs. (13) and (14) have been included with the exception of the separation cut $\left|y_{j 1}-y_{j 2}\right|>$ 4.4. The right upper, left lower, and right lower panels illustrate the effect of the jet-jet invariant mass cut, the lepton rapidity cut, and the rapidity separation between lepton-jet cut, respectively, in reducing the QCD background. In these figures the gap survival probabilities in Eq. (10) are included except for the left upper panel.

invariant mass distribution of the tagging jets and in the lepton rapidity distributions between the QCD and EW backgrounds, as illustrated in the upper right and lower panels of Fig. 1. First, since the QCD distribution exhibits a larger slope than the EW and signal ones in the invariant mass distribution of the tagging jets $\left(M_{j j}\right)$, a hard cut in $M_{j j}$ tends to suppress the QCD background and enhance the signal. Second, as expected, the charged lepton rapidity is larger for the QCD processes since in this case the $W^{+}$production is dominated by the bremsstrahlung of the gauge boson off initial and final state quarks, and,
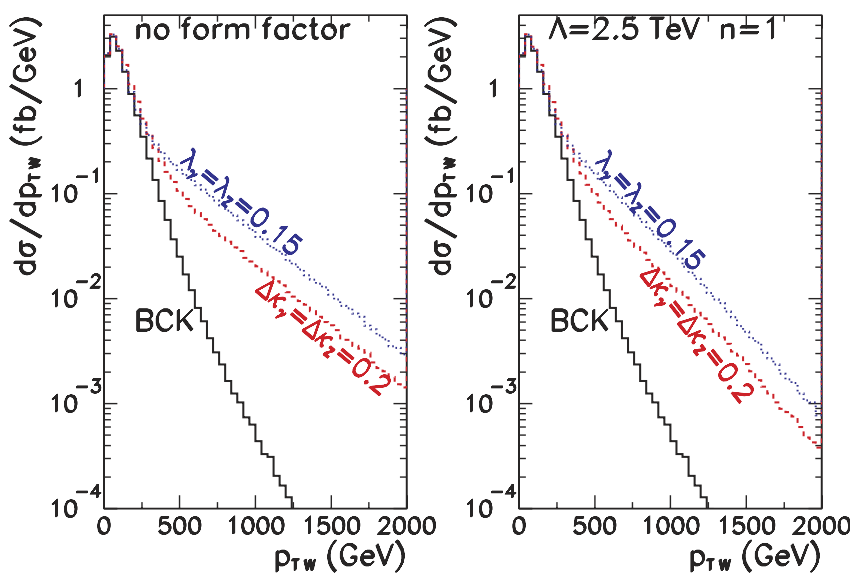

FIG. 2 (color online). Transverse momentum distribution for the $W^{+}$produced in $W^{+} j j$ events with $W^{+} \rightarrow l^{+} \nu_{l}$ at LHC after applying the cuts (13)-(16). The left panel shows the distribution for the background and anomalous TGV signal without the effect of the form factor (11). In the right panel this form factor has been included for $n=1$ and $\Lambda=2.5 \mathrm{TeV}$.

consequently, the charged lepton has a tendency to be closer to the beam pipe or to the tagging jets.

Taking into account all these properties of the signal and backgrounds, we further required

$$
\begin{aligned}
\left|\Delta y_{\ell j}\right| & \geq 2, \quad\left|y_{\ell}\right| \leq 1.5, \\
M_{j j} & \geq \begin{cases}2000 \mathrm{GeV} & \text { for } W^{ \pm} \text {production } \\
1200 \mathrm{GeV} & \text { for } Z \text { production }\end{cases}
\end{aligned}
$$

in order to suppress the QCD background.

With these cuts we have selected the phase space region where WBF processes dominate but so far we have not made any selective cut to discriminate between the anomalous signal and the SM contribution to WBF. In order to do so, we make use of the fact that, due to the presence of operators with higher derivatives and the loss of unitarity, anomalous couplings lead to the enhancement of the transverse momentum distribution of the electroweak bosons at high $p_{T}$ 's as illustrated in Fig. 2 . Therefore, the anomalous signal can be enhanced by requiring that

TABLE II. SM, anomalous, and interference terms as defined in Eq. (12) for $W^{+}$production

\begin{tabular}{|c|c|c|c|c|c|c|c|c|}
\hline \multirow[b]{2}{*}{$\sigma_{W^{+}} \times P_{\text {surv }} \times$ eff $(\mathrm{fb})$} & \multicolumn{3}{|c|}{ SM } & \multicolumn{4}{|c|}{ Anomalous } & \multirow[b]{2}{*}{$g_{5}^{Z}$} \\
\hline & QCD & EW & $\Delta \kappa_{\gamma}$ & $\lambda_{\gamma}$ & $\Delta g_{1}^{Z}$ & $\Delta \kappa_{Z}$ & $\lambda_{Z}$ & \\
\hline SM & 0.71 & 4.70 & 6.5 & -1.2 & 12.0 & 8.8 & -4.4 & 1.0 \\
\hline$\Delta \kappa_{\gamma}$ & & & 49.0 & -7.5 & -17.1 & 70.9 & -4.3 & -1.6 \\
\hline$\lambda_{\gamma}$ & & & $\cdots$ & 323.3 & 8.8 & -4.3 & 354.0 & -1.3 \\
\hline$\Delta g_{1}^{Z}$ & & & $\cdots$ & $\cdots$ & 161.1 & -70.7 & 35.5 & 11.9 \\
\hline$\Delta \kappa_{Z}$ & & & $\ldots$ & $\ldots$ & $\ldots$ & 180.7 & -16.0 & -2.0 \\
\hline$\lambda_{Z}$ & & & $\cdots$ & $\ldots$ & $\ldots$ & $\cdots$ & 735.1 & -6.5 \\
\hline$g_{5}^{Z}$ & & & $\cdots$ & $\cdots$ & $\cdots$ & $\cdots$ & $\ldots$ & 31.5 \\
\hline
\end{tabular}
after applying the cuts (13)-(16). 
TABLE III. SM, anomalous, and interference terms as defined in Eq. (12) for $W^{-}$production after applying the cuts (13)-(16).

\begin{tabular}{|c|c|c|c|c|c|c|c|c|}
\hline \multirow[b]{2}{*}{$\sigma_{W^{-}} \times P_{\text {surv }} \times \operatorname{eff}(\mathrm{fb})$} & \multicolumn{2}{|c|}{ SM } & \multicolumn{6}{|c|}{ Anomalous } \\
\hline & QCD & EW & $\Delta \kappa_{\gamma}$ & $\lambda_{\gamma}$ & $\Delta g_{1}^{Z}$ & $\Delta \kappa_{Z}$ & $\lambda_{Z}$ & $g_{5}^{Z}$ \\
\hline SM & 0.30 & 1.98 & 2.9 & -0.4 & 4.8 & 3.8 & -1.7 & -0.5 \\
\hline$\Delta \kappa_{\gamma}$ & & & 20.5 & -3.2 & -7.2 & 29.1 & -1.4 & 1.1 \\
\hline$\lambda_{\gamma}$ & & & $\cdots$ & 127.8 & 3.5 & -1.4 & 132.2 & -0.8 \\
\hline$\Delta g_{1}^{Z}$ & & & $\ldots$ & $\ldots$ & 64.0 & -29.6 & 15.2 & -2.6 \\
\hline$\Delta \kappa_{Z}$ & & & $\cdots$ & $\cdots$ & $\cdots$ & 74.4 & -6.3 & 3.6 \\
\hline$\lambda_{Z}$ & & & $\cdots$ & $\cdots$ & $\ldots$ & $\ldots$ & 281.2 & -1.7 \\
\hline$g_{5}^{Z}$ & & & $\cdots$ & $\cdots$ & $\cdots$ & $\cdots$ & $\cdots$ & 13.2 \\
\hline
\end{tabular}

$$
p_{T}^{W} \geq 300 \mathrm{GeV}, \quad p_{T}^{Z} \geq 100 \mathrm{GeV} .
$$

One must note, however, that this enhancement must be effectively cut off before it leads to unacceptable violations of unitarity. As discussed in the previous section, we follow the standard procedure to avoid this unphysical behavior of the cross section and multiply the anomalous couplings by a form factor which we chose to be as Eq. (11). The effect of this form factor can be seen by comparing the right and left panels of Fig. 2. It is worth commenting that pair production of gauge bosons is affected by the details of the form factors in a different way and it is probably more sensitive to them [8]. This further stresses the importance of studying both single and double pair production to obtain the most meaningful information on the TGV's.

The final results on the EW and QCD background cross section as well as the anomalous contributions to the coefficients in Eq. (12) after applying the cuts (13)-(16) is presented in Tables II, III, and IV, which already include the effect of veto survival probability and the detection efficiency 0.85 for each charged lepton. From the tables we read that after applying the cuts (13)-(16) about $15 / 85 \%(30 / 70 \%)$ of the background $W^{ \pm}(Z)$ events are due to $\mathrm{QCD} / \mathrm{EW}$ processes. Moreover, we have verified that the $t \bar{t}+n$ jets background is negligible after applying cuts (13) and (14) and vetoing extra jets or leptons in the central rapidity region of the detector.

\section{PREDICTING THE BACKGROUND}

In this work we estimate the LHC potential to constrain anomalous TGV's in $j j \ell^{ \pm} / p_{T}$ and $j j \ell^{+} \ell^{-}$events by considering only the total cross section after cuts; that is, we analyze the sensitivity for TGV's of a counting experiment. The sensitivity of this search is thus determined by the precision with which the background rate in the search region can be predicted.

This is a challenging task, in particular, for the QCD background. Since the signal selection is demanding, including double forward jet tagging and central jet vetoing techniques whose acceptance cannot be calculated with sufficient precision in perturbative QCD, the theoretically predicted background can vary up to a factor of 3 depending on the choices of factorization and renormalization scales. Therefore, the possibility of obtaining meaningful information on deviations from the TGV's is directly limited by our ability to determine the background directly from LHC data.

This background normalization error can be reduced by relaxing some of the cuts, i.e., by considering a larger phase space region as a calibration region. The background expected in the signal region is then obtained by extrapolation of the measured events in the calibration region to the signal region according to perturbative QCD. This procedure introduces also an uncertainty, which we denote as QCD-extrapolation uncertainty, due to the extrapolation to the signal region. However, as we will show, these uncertainties are smaller than the uncertainties for the total cross section.

We defined the calibration region by the cuts (13)-(15) and the requirement that $p_{T}^{W}<250 \mathrm{GeV}$ or $p_{T}^{Z}<100 \mathrm{GeV}$ - that is, we modified only the cut (16) which is intended to enhance the signal. This choice of the calibration region has the virtue of preserving the requirements on the jets and, consequently, not affecting significantly the veto survival probability.

Shown in Fig. 3 is $d \sigma / d p_{T}^{W^{+}}$for four different choices of the renormalization and factorization scales: $\mathbf{C 1}, \mu_{F}^{0}=$ $\mu_{R}^{0}=\sqrt{\left(p_{T j_{1}}^{2}+p_{T j_{2}}^{2}\right) / 2} \quad$ (dashed line); $\mathbf{C 2}, \quad \mu_{R}^{0}=$ $\sqrt{\left(p_{T j_{1}}^{2}+p_{T j_{2}}^{2}\right) / 2}$ and $\mu_{F}^{0}=\sqrt{\hat{s}}$ (dashed-dotted line),

TABLE IV. SM, anomalous, and interference terms as defined in Eq. (12) for $Z$ production after applying the cuts (13)(16).

\begin{tabular}{cccrrrr}
\hline \hline & \multicolumn{3}{c}{$\mathrm{SM}$} & \multicolumn{4}{c}{ Anomalous } \\
$\sigma_{Z} \times P_{\text {surv }} \times$ eff $(\mathrm{fb})$ & $\mathrm{QCD}$ & $\mathrm{EW}$ & $\Delta g_{1}^{Z}$ & $\Delta \kappa_{Z}$ & $\lambda_{Z}$ & $g_{5}^{Z}$ \\
\hline $\mathrm{SM}$ & 1.37 & 3.40 & 9.0 & 0.0 & 0.6 & 0.0 \\
$\Delta g_{1}^{Z}$ & $\ldots$ & 22.7 & 0.2 & -8.7 & 0.0 \\
$\Delta \kappa_{Z}$ & $\ldots$ & $\cdots$ & 8.3 & 11.1 & 0.0 \\
$\lambda_{Z}$ & $\ldots$ & $\ldots$ & $\cdots$ & 52.8 & 0.0 \\
$g_{5}^{Z}$ & $\ldots$ & $\ldots$ & $\cdots$ & $\cdots$ & 13.9 \\
\hline \hline
\end{tabular}




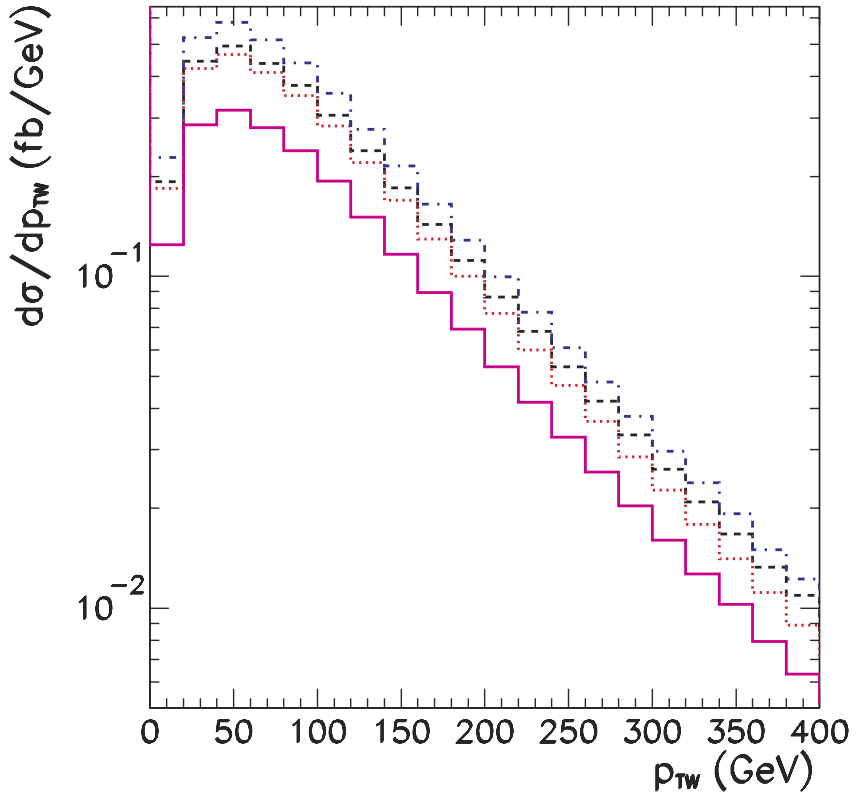

FIG. 3 (color online). $\quad W^{+}$transverse momentum distribution for different choices of the renormalization and factorization scales after cuts (13)-(15). The dashed line stands for $\mu_{F}^{0}=$ $\mu_{R}^{0}=\sqrt{\left(p_{T j_{1}}^{2}+p_{T j_{2}}^{2}\right) / 2}$ and the dashed-dotted line represents $\mu_{R}^{0}=\sqrt{\left(p_{T j_{1}}^{2}+p_{T j_{2}}^{2}\right) / 2}$ and $\mu_{F}^{0}=\sqrt{\hat{s}}$, where $\hat{s}$ is the squared parton center-of-mass energy. Our default choice $\alpha_{s}^{2}\left(\mu_{R}^{0}\right)=$ $\alpha_{s}\left(p_{T j_{1}}\right) \alpha_{s}\left(p_{T j_{2}}\right)$ and $\mu_{F}^{0}=\min \left(p_{T j 1}^{2}, p_{T j 2}^{2}, p_{T W}^{2}\right)$ is represented by the solid line, while the dotted line stands for $\mu_{R}^{0}=$ $\sqrt{p_{T j 1} p_{T j 2}}$ and $\mu_{F}^{0}=\left(p_{T j 1} p_{T j 2} E_{W}^{2}\right)^{1 / 4}$, where $E_{W}^{2}=$ $p_{T W}^{2}+m_{W}^{2}$.

where $\hat{s}$ is the squared parton center-of-mass energy; C3, $\quad \mu_{R}^{0}=\sqrt{p_{T j 1} p_{T j 2}}$ and $\mu_{F}^{0}=\left(p_{T j 1} p_{T j 2} E_{W}^{2}\right)^{1 / 4}$ (dotted line), where $E_{W}^{2}=p_{T W}^{2}+m_{W}^{2}$; $\mathbf{C 4}$, our default choice $\alpha_{s}^{2}\left(\mu_{R}^{0}\right)=\alpha_{s}\left(p_{T j_{1}}\right) \alpha_{s}\left(p_{T j_{2}}\right)$ and $\mu_{F}^{0}=$ $\min \left(p_{T j 1}^{2}, p_{T j 2}^{2}, p_{T W}^{2}\right)$ (solid line). In this figure we have added the electroweak and QCD contributions, taking into account the corresponding veto survival probabilities.

At present we have only leading order (LO) calculations of the $W j j$ and $Z j j$ QCD backgrounds available. Because of the small difference in weak boson mass, as compared to, e.g., the large dijet mass required in our event selection, QCD corrections for these processes are similar and we present only the $p_{T}^{W^{+}}$distribution for the $W^{+} j j$ background in the following, but in our analysis we have evaluated the uncertainties for the processes $W^{+} j j$, $W^{-} j j$, and $Z j j$. As we can see from Fig. 3, the normalization of the background changes by up to a factor of 2 between these choices. Moreover, another variation by a factor of 1.5 is obtained by changing individual renormalization scales between $\mu_{R}=\mu_{R}^{0} / 10$ and $\mu_{R}=10 \mu_{R}^{0}$. However, while the normalization of the $W^{+} j j$ cross section changes drastically, the shape of the $p_{T}^{W^{+}}$distribution is essentially unaffected.

As a measure of shape changes, we study the ratio of the cross sections in the signal region and the calibration region $\left(R_{V}\right.$ for $\left.V=W^{ \pm}, Z\right)$ as a function of $\xi$, the scale factor for the four different renormalization scale choices $\mu_{R}=\xi \mu_{R}^{0}$ listed above. For example, for $W^{+}$we define the ratio of the cross sections obtained, imposing that $p_{T}^{W^{+}}>300 \mathrm{GeV}$ and $p_{T}^{W^{+}}<250 \mathrm{GeV}$,

$$
R_{W^{+}}=\frac{\sigma\left(p_{T}^{W^{+}}>300 \mathrm{GeV}\right)}{\sigma\left(p_{T}^{W^{+}}<250 \mathrm{GeV}\right)},
$$

where, in the evaluation of these ratios, we have added the electroweak and QCD contributions, taking into account the corresponding veto survival probabilities. The $\xi$ dependence shown in Fig. 4 is small for individual choices of $\mu_{R}^{0}$, being smaller than the differences between the four basic choices for $\mu_{R}^{0}$ and $\mu_{F}^{0}$. Furthermore, this figure also illustrates the effect of changing the factorization scale as well. For instance, the dashed and the dotted-dashed lines differ only by the choice of the factorization scale, allowing us to see that different choices in the factorization or renormalization scales lead to similar modifications of the predictions.



FIG. 4 (color online). The ratio $R_{W^{+}}$is shown as a function of $\xi$, where $\mu_{R}=\xi \mu_{R}^{0}$ and different choices of $\mu_{R}^{0}$ and $\mu_{F}^{0}$. The dashed line stands for $\mu_{F}^{0}=\mu_{R}^{0}=\sqrt{\left(p_{T j_{1}}^{2}+p_{T j_{2}}^{2}\right) / 2}$ and the dashed-dotted line represents $\mu_{R}^{0}=\sqrt{\left(p_{T j_{1}}^{2}+p_{T j_{2}}^{2}\right) / 2}$ and $\mu_{F}^{0}=\sqrt{\hat{s}}$, where $\hat{s}$ is the squared parton center-of-mass energy. Our default choice $\alpha_{s}^{2}\left(\mu_{R}^{0}\right)=\alpha_{s}\left(p_{T j_{1}}\right) \alpha_{s}\left(p_{T j_{2}}\right)$ and $\mu_{F}^{0}=$ $\min \left(p_{T j 1}^{2}, p_{T j 2}^{2}, p_{T W}^{2}\right)$ is represented by the solid line, while the dotted line stands for $\mu_{R}^{0}=\sqrt{p_{T j 1} p_{T j 2}}$ and $\mu_{F}^{0}=$ $\left(p_{T j 1} p_{T j 2} E_{W}^{2}\right)^{1 / 4}$, where $E_{W}^{2}=p_{T W}^{2}+m_{W}^{2}$. 
Figure 4 can be used as a good estimate of the uncertainty associated with the choice of scales due to the different choices used in it. From this figure it is clear that the extrapolation uncertainty in this case is rather small $(\simeq$ $7 \%$ ) despite the use of a LO QCD calculation. Inclusion of next-to-leading-order (NLO) calculation should reduce this shape uncertainty. This reduction is indeed observed once NLO QCD radiative corrections are included in the QCD contribution [9] and in the EW contribution [10], respectively. Once the combined calculation including QCD radiative corrections to QCD, EW, and anomalous contributions becomes available, it will be possible to have a full NLO estimate of the background uncertainties and the expected signals.

Altogether, the total expected uncertainty in the estimated number of background events has two sources: the theoretical uncertainty associated to the extrapolations from the calibration region $\left(\delta_{\text {bck,th }}\right)$ and the statistical error associated to the determination of the background cross section in the calibration region $\left(\delta_{\text {bck,stat }}\right)$. We estimated the theoretical extrapolation error from the results obtained using our four basic choices for $\mu_{R}^{0}$ and $\mu_{F}^{0}$. If $R_{\max (\min )}$ is the largest (smallest) value for $R_{W^{+}}$using our four basic choices of scales, we defined

$$
\delta_{\mathrm{bck}, \mathrm{th}}=\frac{R_{\max }-R_{\min }}{R_{\max }+R_{\min }} .
$$

Table $\mathrm{V}$ exhibits our results for these errors as relative uncertainties, assuming an integrated luminosity of $100 \mathrm{fb}^{-1}$. As we can see from this table, the theoretical extrapolation error is the largest uncertainty.

\section{RESULTS AND DISCUSSION}

In order to extract the attainable limits on the anomalous TGV's, we assumed an integrated luminosity of $100 \mathrm{fb}^{-1}$ and that the observed number of events $V j j$ with $V=W^{ \pm}, Z$ is compatible with the SM expectations for the choice $\mathbf{C} \mathbf{4}$ of the renormalization and factorization scales both in the signal $\left(N_{\mathrm{V} \text {,data }}^{\mathrm{S}}\right)$ and in the calibration $\left(N_{\mathrm{V}, \text { data }}^{\mathrm{C}}\right)$ regions, i.e.,

$$
N_{\mathrm{V}, \text { data }}^{\mathrm{S}}=N_{\mathrm{V}, \mathrm{SM}, \mathrm{C} 4}^{\mathrm{S}} \quad \text { and } \quad N_{\mathrm{V}, \text { data }}^{\mathrm{C}}=N_{\mathrm{V}, \mathrm{SM}, \mathrm{C} 4}^{\mathrm{C}} .
$$

We also assumed no charge discrimination, and, consequently, we combined $W^{+} j j$ and $W^{-} j j$ events.

TABLE V. Uncertainties of the background estimate. The statistical error was estimated assuming an integrated luminosity of $100 \mathrm{fb}^{-1}$.

\begin{tabular}{ccc}
\hline \hline Final state & $\delta_{\text {bck,stat }}(\%)$ & $\delta_{\text {bck,th }}(\%)$ \\
\hline$W^{+} j j$ & 0.90 & 7.4 \\
$W^{-} j j$ & 1.3 & 5.7 \\
$W^{ \pm} j j$ & 0.74 & 4.4 \\
$Z j j$ & 5.4 & 2.6 \\
\hline \hline
\end{tabular}

The anomalous TGV's manifest themselves as a difference between the number of observed events and the number of background events estimated from the extrapolation of the background measured in the calibration region $\left(N_{\mathrm{V}, \text { back }}^{\mathrm{S}}\right)$; that is,

$$
N_{\mathrm{V}, \text { data }}^{\mathrm{S}}-N_{\mathrm{V}, \text { back }}^{\mathrm{S}}
$$

where $N_{\mathrm{V}, \text { back }}^{\mathrm{S}}=R_{V} N_{\mathrm{V}, \text { data }}^{\mathrm{C}}$. The statistical error of the number of anomalous events is

$$
\sigma_{\text {stat }}^{2}=N_{\mathrm{V}, \text { data }}^{\mathrm{S}}+\left(R_{V} N_{\mathrm{V}, \text { data }}^{\mathrm{C}} \delta_{\text {bck,stat }}\right)^{2},
$$

where the first term is the statistical error of the measured number of events in the signal region and the second term is the error in the determination of the background in the signal region due to the statistical error of the background measurement in the calibration region, $\delta_{\text {bck,stat }}$. Both errors can be assumed to be Gaussian and we combine them in quadrature.

So at $95 \%$ confidence level (CL) we can impose a limit on the anomalous couplings from the condition

$$
\begin{aligned}
& \left|N_{V}\left(g_{\text {ano }}\right)-N_{\mathrm{V}, \text { data }}^{\mathrm{S}}+N_{\mathrm{V}, \text { back }}^{\mathrm{S}}\right| \\
& \quad=\left|N_{V}\left(g_{\text {ano }}\right)-N_{\mathrm{V}, \text { data }}^{\mathrm{S}}+R_{V} N_{\mathrm{V}, \text { data }}^{\mathrm{C}}\right| \leq 1.96 \sigma_{\text {stat }},
\end{aligned}
$$

where $N_{V}\left(g_{\text {ano }}\right)$ stands for the expected number of anomalous events that can be inferred using Eq. (12). From Eq. (20) it is clear that the extracted bound on the anomalous couplings depends on what we assume for the range of $R_{V}$ compatible with the measured background in the calibration region. In other words, the constraints depend on how much of the estimated range for the number of background events due to the extrapolation uncertainty will still be allowed once the measurement in the calibration region is available.

As a benchmark, we first evaluate the attainable $95 \%$ CL constraints on the TGV's, neglecting the extrapolation uncertainty; that is, for $R_{V}=N_{\mathrm{V}, \text { data }}^{\mathrm{S}} / N_{\mathrm{V} \text {,data }}^{\mathrm{C}}$. In this case and assuming that only one TGV is nonvanishing, we get

$$
\begin{aligned}
-0.18 \leq \Delta \kappa_{\gamma} \leq 0.045, & -0.033 \leq \lambda_{\gamma} \leq 0.037, \\
-0.075 & \leq \Delta g_{1}^{Z} \leq 0.023, \quad-0.077 \leq \Delta \kappa_{Z} \leq 0.029, \\
-0.021 \leq \lambda_{Z} \leq 0.027, & -0.12 \leq g_{5}^{Z} \leq 0.10 .
\end{aligned}
$$

We also varied the cuts (13)-(16) in order to verify whether these limits could be improved. Nevertheless, it turns out that these are the best bounds except for $\kappa_{\gamma}$, which is better constrained when we relax the following cuts $^{2}$ :

$$
M_{j j}>1200 \mathrm{GeV} \quad \text { and } \quad p_{T}^{W}>100 \mathrm{GeV},
$$

\footnotetext{
${ }^{2}$ In this case we define the calibration region with $M_{j j}>$ $1200 \mathrm{GeV}$ and $p_{T}^{W}<100 \mathrm{GeV}$ and determine the corresponding uncertainties.
} 
leading to

$$
-0.036 \leq \Delta \kappa_{\gamma} \leq 0.031
$$

We learn from these results that the analysis of the total number of events for single production of electroweak gauge bosons has the potential of improving the constraints with respect to the pair production for the couplings $\Delta \kappa_{\gamma}$ and $\Delta \kappa_{Z}$; see Table I.

Next we conservatively estimate the $95 \%$ CL sensitivity limits at the LHC assuming the largest (or smallest) possible background within the full range of our presently estimated LO extrapolation uncertainty $R_{V}=$ $N_{\mathrm{V}, \text { data }}^{\mathrm{S}} / N_{\mathrm{V}, \text { data }}^{\mathrm{C}}\left(1 \pm \delta_{\text {back,th }}\right)$. In this case the most conservative $95 \%$ CL bound can be obtained from

$$
\begin{aligned}
\left|N_{V}\left(g_{\text {ano }}\right)\right| & \leq\left|N_{\mathrm{V}, \text { data }}^{\mathrm{S}}-R_{V} N_{\mathrm{V}, \text { data }}^{\mathrm{C}}\right|_{\max }+1.96 \sigma_{\text {stat }} \\
& \leq N_{\mathrm{V}, \text { data }}^{\mathrm{S}} \delta_{\text {bck,th }}+1.96 \sigma_{\text {stat }},
\end{aligned}
$$

which leads to

$$
\begin{aligned}
-0.20 \leq \Delta \kappa_{\gamma} \leq 0.065, & -0.043 \leq \lambda_{\gamma} \leq 0.046, \\
-0.097 \leq \Delta g_{1}^{Z} \leq 0.035, & -0.089 \leq \Delta \kappa_{Z} \leq 0.039, \\
-0.027 & \leq \lambda_{Z} \leq 0.033, \quad-0.14 \leq g_{5}^{Z} \leq 0.13 .
\end{aligned}
$$

Last we estimate the attainable $95 \%$ CL constraints at the LHC, assuming that the extrapolation uncertainty will be reduced by a factor of 2 once NLO predictions are available and/or the data from the calibration region reduces the allowed range of background predictions:

$$
\begin{aligned}
& -0.066 \leq \Delta \kappa_{\gamma} \leq 0.052, \quad-0.038 \leq \lambda_{\gamma} \leq 0.042, \\
& -0.086 \leq \Delta g_{1}^{Z} \leq 0.029, \quad-0.083 \leq \Delta \kappa_{Z} \leq 0.034, \\
& -0.024 \leq \lambda_{Z} \leq 0.030, \quad-0.13 \leq g_{5}^{Z} \leq 0.12,
\end{aligned}
$$

where the bounds on $\Delta \kappa_{\gamma}$ have been obtained with the relaxed cuts in Eq. (22).

In deriving these bounds, we have statistically combined the results from $W^{ \pm} j j$ and $Z j j$ production although the limits originate basically from $W^{ \pm} j j$ production, having the $Z j j$ process a marginal impact on the constraints due to the small SM-anomalous interference cross section; see Table IV. The only anomalous couplings for which the $Z j j$ production plays any role is $g_{1}^{Z}$. Moreover, the presence of nonvanishing interference between the SM and anomalous contributions lead to bounds that are asymmetrical around zero.

We can see from Tables II, III, and IV that there is nonvanishing interference between the different anomalous TGV's contributions, and, consequently, there will be nontrivial correlations between the bounds on these couplings when more than one coupling is nonvanishing. We depict in Fig. 5 the 95\% CL ( 2 degrees of freedom) regions in the planes $\left(\Delta g_{1}^{Z}, \Delta \kappa_{Z}\right)$ and $\left(\lambda_{Z}, \lambda_{\gamma}\right)$. As we can see from this figure there is correlation between $\left(\Delta g_{1}^{Z}, \Delta \kappa_{Z}\right)$ and anticorrelation between $\left(\lambda_{Z}, \lambda_{\gamma}\right)$.
In the framework of effective Lagrangians exhibiting a linear realization of the $\mathrm{SU}(2)_{X} \otimes \mathrm{U}(1)_{Y}$ symmetry, the anomalous TGV's satisfy the relations (5)-(7). Assuming these constraints among the anomalous interactions and $f_{B}=f_{W}$, the potential LHC 95\% CL bounds are

$$
\begin{aligned}
& -0.052(-0.034) \leq \Delta \kappa_{\gamma} \leq 0.040(0.028), \\
& -0.019(-0.017) \leq \lambda_{\gamma(Z)} \leq 0.023(0.021), \\
& -0.097(-0.090) \leq \Delta g_{1}^{Z} \leq 0.019(0.016), \\
& -0.052(-0.049) \leq \Delta \kappa_{Z} \leq 0.010(0.0085),
\end{aligned}
$$

when we use the full (half) LO extrapolation uncertainty and the relaxed cuts for $\Delta \kappa_{\gamma}$. The constraints on $g_{5}^{Z}$ are the ones given in Eqs. (25) and (26) since $g_{5}^{Z}$ is not related to the other couplings in the HISZ scenario.

The improvement of the constraints on $\lambda_{\gamma(Z)}$ in the HISZ scenario is easy to understand by remembering that the TGV's $\lambda_{Z}$ and $\lambda_{\gamma}$ are anticorrelated as shown in Fig. 5. On the other hand, the tighter bounds obtained for $\Delta \kappa_{\gamma}, g_{1}^{Z}$, and $\Delta \kappa_{Z}$ originate from the hypothesis $f_{W}=$ $f_{B}$, which relates these couplings, leading to just one independent combination of them.

We can learn from the above results that the study of the production rates of single electroweak gauge bosons via $\mathrm{WBF}$ at the LHC can lead to upper bounds on $\Delta \kappa_{Z}$ that are of the same order of the bounds derived from the kinematical analysis of pair production of electroweak gauge bosons. In the case of the anomalous coupling $\Delta \kappa_{\gamma}$, the upper bound can be slightly weaker or of the same order as that coming from gauge-boson pair production. On the other hand, the bounds on $\lambda_{Z, \gamma}$ and $g_{1}^{Z}$ obtained via the latter process are more stringent.

There is still room for further improvements in our analyses, which are beyond the scope of this work. First, the importance of the single $Z$ production can be enlarged by considering the invisible decay of the $Z$ into neutrino pairs. This process can certainly be extracted from the backgrounds analogously to the case of invisibly decaying Higgs bosons [24]. Second, the use of higher order QCD calculations might lead to lower extrapolation errors from the calibration regions as illustrated above. Probably the most significant improvement will be the


FIG. 5. $95 \% \mathrm{CL}$ allowed region in the planes $\left(\Delta g_{1}^{Z}, \Delta \kappa_{Z}\right)$ and $\left(\lambda_{Z}, \lambda_{\gamma}\right)$. 


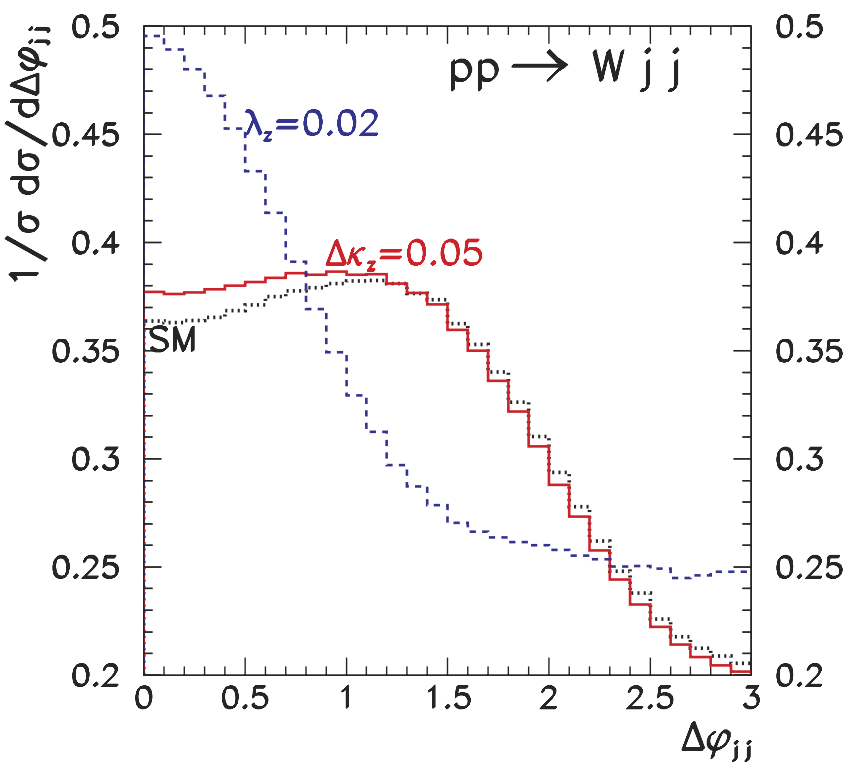

FIG. 6 (color online). Normalized $\Delta \varphi_{j j}$ distribution for $W^{+} j j$. The dotted line stands for the SM result while the dashed (solid) line stands for the resulting assuming $\lambda_{Z}=$ $0.02\left(\Delta \kappa_{Z}=0.05\right)$.

use of kinematical distributions to constrain the anomalous TGV's in analogy with what is done in the analysis of electroweak gauge-boson pair production [6]. Notice that with the cuts proposed the final data sample contains $\sim 770$ electroweak $W^{ \pm}$events and $\sim 480 \mathrm{Z}$ events for an integrated luminosity of $\mathcal{L}=100 \mathrm{fb}^{-1}$, allowing for statistically meaningful binning. In general, the anomalous couplings can be better constrained by fitting the electroweak vector-boson transverse momentum distribution; see Fig. 2. The anomalous couplings $\lambda_{Z, \gamma}$ can also be better determined by studying the angle in the transverse plane between the tagging jets $\left(\Delta \varphi_{j j}\right)$. In fact, Fig. 6 shows that the shape of the $\Delta \varphi_{j j}$ distribution is quite different for these couplings when compared to the SM predictions or the other anomalous contributions. Therefore, should an event excess be found, this distribution can be used to discriminate among the different couplings.

In summary, we have shown that the single production of electroweak gauge bosons via the weak boson fusion processes at LHC can provide stringent tests on the deviations of the TGV's from the standard model prediction. This is possible because the QCD background can be efficiently reduced by exploiting the difference in several kinematical distributions. Furthermore, we have shown that the background can be estimated with good enough precision by extrapolation from the measured rate in a signal-suppressed calibrating region of the phase space. Altogether the sensitivity bounds obtained for some of the anomalous couplings are comparable to those attainable from the study of electroweak gauge-boson pair production. With these results we stress the importance of studying both types of reaction to obtain maximum information on the gauge structure of the electroweak interactions.

\section{ACKNOWLEDGMENTS}

We thank J. Hobbs, M. Maltoni, and R. ZukanovichFunchal for very useful discussions and comments. M. C. G.-G. thanks IF-USP, while O. É. thanks Y.I.T.P. for their hospitality during the development of most of this work. This work was supported by Conselho Nacional de Desenvolvimento Científico e Tecnológico (CNPq), by Fundação de Amparo à Pesquisa do Estado de São Paulo (FAPESP), and by Programa de Apoio a Núcleos de Excelência (PRONEX). M. C. G.-G. acknowledges support from National Science Foundation Grant No. PHY0098527 and Spanish Grants No. FPA-20013031 and No. CTIDIB/2002/24.
[1] For a review, see H. Aihara et al., in Electroweak Symmetry Breaking and New Physics at the TeV Scale, edited by T. Barklow, S. Dawson, H. Haber, and J. Seigrist (World Scientific, Singapore, 1996), p. 488.

[2] CDF Collaboration, K. Gounder et al., hep-ex/9903038; D0 Collaboration, B. Abbott et al., Phys. Rev. D 62, 052005 (2000).

[3] L3 Collaboration, P. Achard et al., Phys. Lett. B 547, 151 (2002); DELPHI Collaboration, P. Abreu et al., Phys. Lett. B 502, 9 (2001); ALEPH Collaboration, A. Heister et al., Eur. Phys. J. C 21, 423 (2001); OPAL Collaboration, G. Abbiendi et al., Eur. Phys. J. C 33, 463 (2004)
[4] ALEPH, DELPHI, L3, OPAL, and SLD Collaborations, LEP Electroweak Working Group, and SLD Heavy Flavor Group, hep-ex/0212036.

[5] S. Alam, S. Dawson, and R. Szalapski, Phys. Rev. D 57, 1577 (1998); S. Dawson and G. Valencia, Phys. Lett. B 333, 207 (1994).

[6] See S. Haywood et al., hep-ph/0003275; G. Azuelos et al., Report No. ATL-PHYS-2001-002; T. Müller, D. Neuberger, and W. H. Thümmel, CMS Note 2000/017, 2000.

[7] U. Baur and D. Zeppenfeld, Nucl. Phys. B308, 127 (1988); U. Baur, T. Han, and J. Ohnemus, Phys. Rev. D 48, 5140 (1993); 57, 2823 (1998); 53, 1098 (1996). 
[8] U. Baur and D. Zeppenfeld, in Workshop on Physics at Current Accelerators and the Supercollider, Argonne, IL, 1993, edited by J.L. Hewett, A. R. White, and D. Zeppenfeld (Argonne National Laboratory, Argonne, IL, 1993), pp. 327-334.

[9] J. Campbell, R. K. Ellis, and D. L. Rainwater, Phys. Rev. D 68, 094021 (2003); J. Campbell and R. K. Ellis, Phys. Rev. D 65, 113007 (2002).

[10] C. Oleari and D. Zeppenfeld, Phys. Rev. D 69, 093004 (2004).

[11] V. D. Barger, K. m. Cheung, T. Han, J. Ohnemus, and D. Zeppenfeld, Phys. Rev. D 44, 1426 (1991); D. L. Rainwater and D. Zeppenfeld, J. High Energy Phys. 9712, 005 (1997); D. L. Rainwater, D. Zeppenfeld, and K. Hagiwara, Phys. Rev. D 59, 014037 (1999); D. L. Rainwater and D. Zeppenfeld, Phys. Rev. D 60, 113004 (1999); 61, 099901(E) (2000).

[12] K. Gaemers and G. Gounaris, Z. Phys. C 1, 259 (1979); K. Hagiwara, K. Hikasa, R. D. Peccei, and D. Zeppenfeld, Nucl. Phys. B282, 253 (1987).

[13] C. P. Burgess and D. London, Phys. Rev. D 48, 4337 (1993); A. F. Falk, M. E. Luke, and E. H. Simmons, Nucl. Phys. B365, 523 (1991).
[14] W. Buchmüller and D. Wyler, Nucl. Phys. B268, 621 (1986).

[15] K. Hagiwara, S. Ishihara, R. Szalapski, and D. Zeppenfeld, Phys. Lett. B 283, 353 (1992); Phys. Rev. D 48, 2182 (1993).

[16] A. De Rujula, M. B. Gavela, P. Hernandez, and E. Masso, Nucl. Phys. B384, 3 (1992).

[17] O. J. P. Éboli, S. M. Lietti, M. C. Gonzalez-Garcia, and S. F. Novaes, Phys. Lett. B 339, 119 (1994).

[18] V. Barger, T. Han, J. Ohnemus, and D. Zeppenfeld, Phys. Rev. Lett. 62, 1971 (1989); Phys. Rev. D 40, 2888 (1989).

[19] H. Chehime and D. Zeppenfeld, Phys. Rev. D 47, 3898 (1993); D. Rainwater, R. Szalapski, and D. Zeppenfeld, Phys. Rev. D 54, 6680 (1996).

[20] T. Stelzer and W. F. Long, Comput. Phys. Commun. 81, 357 (1994).

[21] CTEQ Collaboration, H. L. Lai et al., Eur. Phys. J. C 12, 375 (2000).

[22] V. Barger, R. Phillips, and D. Zeppenfeld, Phys. Lett. B 346, 106 (1995).

[23] D. Rainwater, hep-ph/9908378.

[24] O. Éboli and D. Zeppenfeld, Phys. Lett. B 495, 147 (2000). 